\title{
OCULOKINETIC PERIMETRY COMPARED WITH HUMPHREY VISUAL FIELD ANALYSIS IN THE DETECTION OF GLAUCOMATOUS VISUAL FIELD LOSS
}

\author{
PETER K. WISHART \\ Liverpool
}

\begin{abstract}
SUMMARY
The ability of oculokinetic perimetry (OKP) to detect glaucomatous central visual field loss was assessed by comparison with the results of Humphrey visual field analysis (HVFA) in a group of patients attending a glaucoma clinic. Fifty-six patients with stable visual fields and good reliability indices on previous automated perimetry examinations underwent programmes 24-1 or 24-2 of the Humphrey visual field analyser and the results compared with those of a field test using a hand-held OKP chart. Forty-three patients had a glaucomatous visual field defect demonstrated by HVFA, and 35 showed a defect to OKP. Thirteen patients were shown to have normal fields on HVFA, and in 21 patients the OKP test was normal. Comparison of the two test results showed that of the 35 patients-with abnormal OKP tests, in 26 the result was a true positive (HVFA also abnormal) and in 9 a false positive (HVFA normal). In the 21 patients with negative OKP tests the results were judged false negatives in $\mathbf{1 3}$ (HFVA showed glaucomatous visual field loss) while in 8 they were true negatives (HVFA normal). Therefore the sensitivity of OKP for the detection of glaucomatous visual field loss was $60.5 \%$ and the specificity for the identification of individuals with glaucomatous visual field loss was $61.5 \%$. In the 26 patients with true positive results there were 36 eyes with positive $\mathrm{OKP}$ charts corresponding to defects on HVFA. Correlation of the number of defects on OKP with the mean deviation and corrected pattern standard deviation of their respective HVFA charts showed a near linear correlation. However, when between one and three points were missed on OKP this loss was equally likely to represent a false positive result as it was to represent the presence of glaucomatous field loss. Analysis of the 26 true positive patients' fields showed that a point missed on OKP corresponded to an average depression of retinal sensitivity of 20.8 decibels on HVFA. However, in $50 \%$ of these patients' fields OKP also failed to detect defects of, on average, 19.6 decibels in another quadrant of the field. This study shows that OKP

Correspondence to: P. K. Wishart, FCOphth, St. Paul's Eye Unit, Royal Liverpool University Hospital, Prescot St., Liverpool L7 8XP, UK.
\end{abstract}

can detect relatively advanced glaucomatous visual field loss, but the low sensitivity and specificity of the test makes this device unsuitable for glaucoma screening.

Oculokinetic perimetry (OKP) is a relatively quick and inexpensive method of testing the central $16^{\circ}$ of the visual field and its use has been suggested as a perimetric technique suitable for glaucoma screening programmes. ${ }^{1.2}$ The unsuitability of performing tonometry alone ${ }^{3}$ and the difficulties of bringing accurate ophthalmoscopy for optic disc evaluation to a community screening programme encourage the use of visual field analysis as the optimum screening method for the detection of individuals who may be suffering from open angle glaucoma. ${ }^{4}$ To be effective in a screening programme, any device must exhibit a high level of sensitivity (ability to detect individuals with the disease) and a high level of specificity (ability to identify normals as such). ${ }^{5}$ The aim of this study was to assess the sensitivity and specificity of OKP in detecting glaucomatous visual field loss by comparing the results with Humphrey visual field analysis (HVFA).

\section{PATIENTS AND METHODS}

Patients who were familiar with HVFA automatic perimetry and were attending the Glaucoma Unit of St. Paul's Eye Hospital, Liverpool, with a diagnosis of glaucoma or ocular hypertension, or who were normotensive with suspicious discs, underwent OKP testing of their visual field. All patients selected were known to be reliable witnesses as in previous field testing their reliability indices (judged by fixation losses, false positive and false negative responses) were within the accepted limits defined by the HVFA's software. ${ }^{6}$ All patients had undergone at least two prior threshold testing visual field examinations of their central visual field with good reliability indices. Any patient with visual acuity less than $6 / 12$ due to media opacities was excluded.

HVFA was performed in most cases using the 24-2 programme with the Statpac 2 analysis of the results pres- 


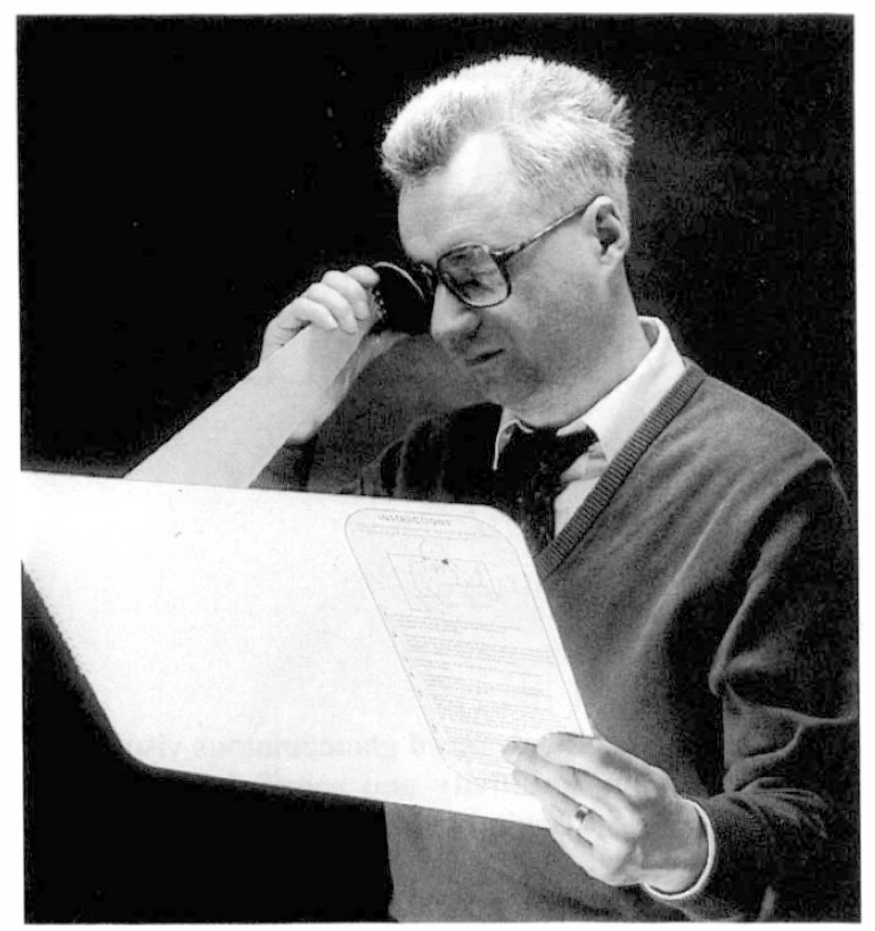

Fig. 1. Hand-held OKP chart in use.

enting the global indices ${ }^{7}$ for each field. For each $24-2$ test a Glaucoma Hemifield Test (GHT) result is automatically provided which states whether the field is 'within normal limits' or shows glaucomatous visual field loss and is 'outside normal limits'. ${ }^{6}$ An objective report on the normality of the field is thus provided, and the clinician does not have to make a subjective judgement on the significance of any departure from normal values which may appear in the field. ${ }^{8}$ The GHT is based on the pattern deviation from normal for an age-matched population on differences of probability scores across the horizontal field meridian. ${ }^{6} \mathrm{~A}$ minority of the patients (known glaucoma cases) had had repeated 24-1 field tests previously and so as not to change the parameters of their follow-up they had a 24-1 test performed for comparison with the OKP test.

The OKP test chart was the hand-held chart described

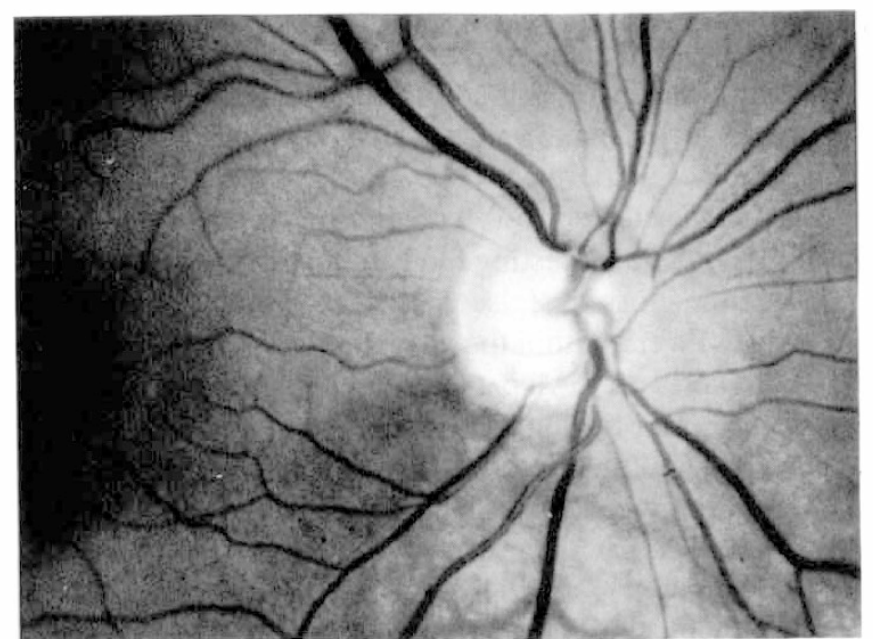

by Damato et al. $^{1}$ and distributed by Merck Sharp and Dohme Ltd. This chart has the advantage over previous designs for OKP testing in that the chart is easily portable and the working distance is kept constant by the fixed sidearm (Fig. 1). The test was carried out in accordance with standard OKP testing technique ${ }^{1}$ in a well-lit room. If glasses were not normally worn for reading, no additional near correction was provided; those patients who normally wore reading glasses had the test conducted wearing their usual glasses. The correct position for the chart was found by confirming the presence of the blind spot for each eye. Then, the patient was instructed to say whether the central target was still visible when looking at each of the 26 test numbers in turn. Any number missed was underlined by the examiner on the score sheet, and the field was retested. Any point missed on this second inspection was marked, and only if the point was missed twice was that point regarded as a defect (positive result). This OKP test chart was compared with a further Humphrey 24-1 or 24-2 central field examination performed within 2 weeks of OKP testing. For HVFA testing, refractive error was corrected using wide-aperture trial lenses with the appropriate reading correction determined by age and current refraction.

OKP testing was carried out by a field technician unaware of the results of the previous HVFA, and similarly the subsequent HVFA was performed independently of the results of the OKP test.

Abnormality of Humphrey visual field was determined by the $\mathrm{GHT}^{6}$ in the case of the 24-2 fields; in the 9 patients undergoing a 24-1 test, a significant abnormality was taken as three or more contiguous points depressed by 6 decibels $(\mathrm{dB})$ or more in a typical location, or one nonedge point depressed by more than $20 \mathrm{~dB} .{ }^{9}$ If any field loss on the latest HVFA did not correspond to that previously identified by HVFA, the test was repeated to confirm its authenticity. Similarly, if OKP testing showed a defect and HVFA was normal, the HVFA was repeated. All HVFA threshold charts supply 'global indices' calculated from the results of the 350 or so questions asked per eye tested and provide for each field the mean deviation (MD) from

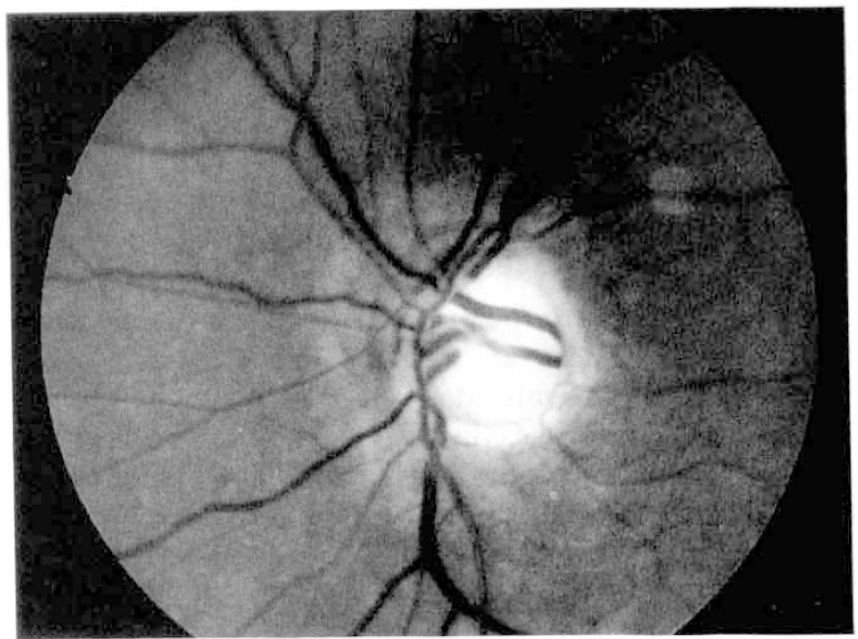

Fig. 2. Optic discs of study patient showing typical glaucomatous damage. Right disc (a) shows loss of a portion of inferior rim. Lef disc (b) shows advanced cupping with loss of temporal and inferior neuroretinal rim. 


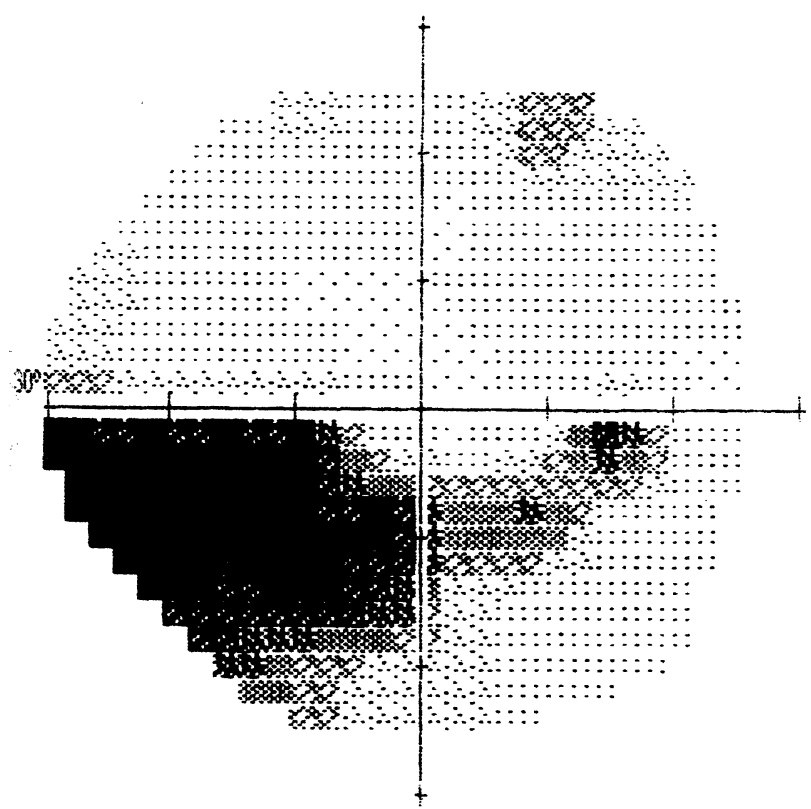

created by the horizontal and vertical meridians crossing at fixation. Any defect identified in one quadrant of the OKP chart was judged real if there was any defect in the corresponding quadrant of the HVFA chart.

All patients had the appearance of their optic disc assessed by the author using stereoscopic slitlamp biomicroscopy with the Volk 78 dioptre lens, and the appearance of the disc was recorded in the notes with a drawing. Many patients also had stereo disc photographs taken. Severity of disc damage was noted, with particular attention to features of glaucomatous disc damage always associated with field loss (obliteration of a portion of the disc rim) or usually associated with visual field loss (pallor and
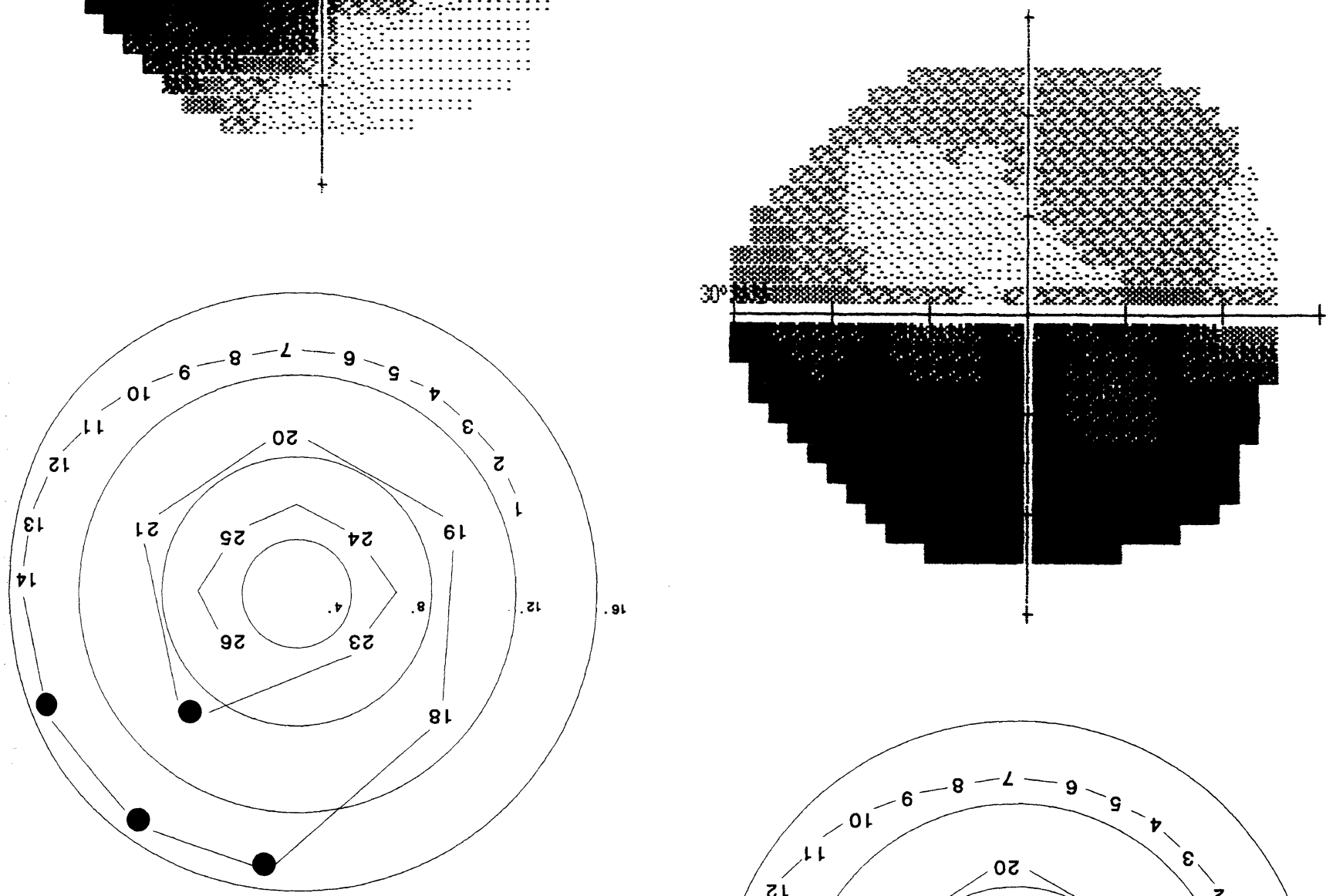

Fig. 3. True positive OKP: grey scale of HVFA and corresponding defect shown by OKP in right eye.

normal, the short-term fluctuation (SF), the pattern deviation (PD) from normal and the corrected pattern standard deviation (CPSD). ${ }^{6}$ The MD gives a mean of the total departure of the field from normality and corresponds to total (overall) field loss. ${ }^{6}$ The CPSD takes into account any short-term fluctuation in the field test and a high CPSD value will identify localised abnormality of the field. Thus a dense localised scotoma will give an abnormally high CPSD value, even if the relative normality of the rest of the field results in only slight overall depression of the total field with consequently a MD within normal limits. ${ }^{7}$

The OKP and HVFA charts were compared with each ther with reference to the four quadrants of the field 
Table I. Visual field status of patients as shown by different methods of perimetry: Humphrey visual field analysis (HVFA) and oculokinetic perimetry (OKP)

\begin{tabular}{lc}
\hline Field & No. of patients \\
\hline Normal & \\
HVFA & 13 \\
OKP & 21 \\
Abnormal & \\
HVFA & 43 \\
OKP & 35 \\
\hline
\end{tabular}

thinning of the rim, disc haemorrhage, vertical elongation of the cup ${ }^{10}$ ) (Fig. 2).

To determine the sensitivity and specificity of OKP as a screening device analysis of the results was performed firstly regarding patients, rather than eyes, as a positive response in one eye identifies a patient as glaucomatous and would direct the patient for further ophthalmic assessment. ${ }^{1}$ The study design also allowed an accurate assessment of the degree of visual field loss that the presence of a defect on OKP testing (positive result) indicates. As HVFA measures the retinal sensitivity, any defect identified by OKP can then be correlated with the HVFA decibel defect identified, and this correlation is presented from corresponding abnormal fields, rather than patients.

\section{RESULTS}

Fixty-six patients were enrolled in the study, with an equal sex distribution of 28 male and 28 female patients. The age range was from 27 years to 82 years with a mean of 60.6 years. Fifty-one patients had OKP and HVFA testing of both eyes; 4 patients were blind in the fellow eye and 1 patient, despite good visual acuity, was unable to find the blind spot with the OKP chart in one eye and could not perform the test in that eye. In 90 of the 107 eyes that underwent both tests, the visual acuity was $6 / 9$ or better. Two eyes were amblyopic but performed equally well with OKP and HVFA and were therefore included in the results.

\section{Sensitivity and Specificity of OKP Testing}

In this section all the results refer to patients, with the more affected eye (worse eye) representing the patient. Table I shows the number of patients identified as having visual field loss with the two methods of field testing. Thirty-five patients had a positive result to OKP testing (i.e. one or more points (numbers) missed) and 21 patients had a negative result to OKP testing (i.e. central target always visible when looking at each test location).

Of the 35 patients with positive OKP tests, in 26 HVFA

Table II. Age (in years) and sex distribution of patients with OKP results judged 'true' (correlating with HVFA) or 'false' (no correlation with HVFA)

\begin{tabular}{lrccl}
\hline & Male & Female & Age range & Mean \\
\hline True positive & 11 & 15 & $27-80$ & 59 \\
False positive & 4 & 5 & $54-68$ & 60.4 \\
True negative & 6 & 2 & $52-73$ & 62 \\
False negative & 7 & 6 & $39-82$ & 61 \\
\hline
\end{tabular}
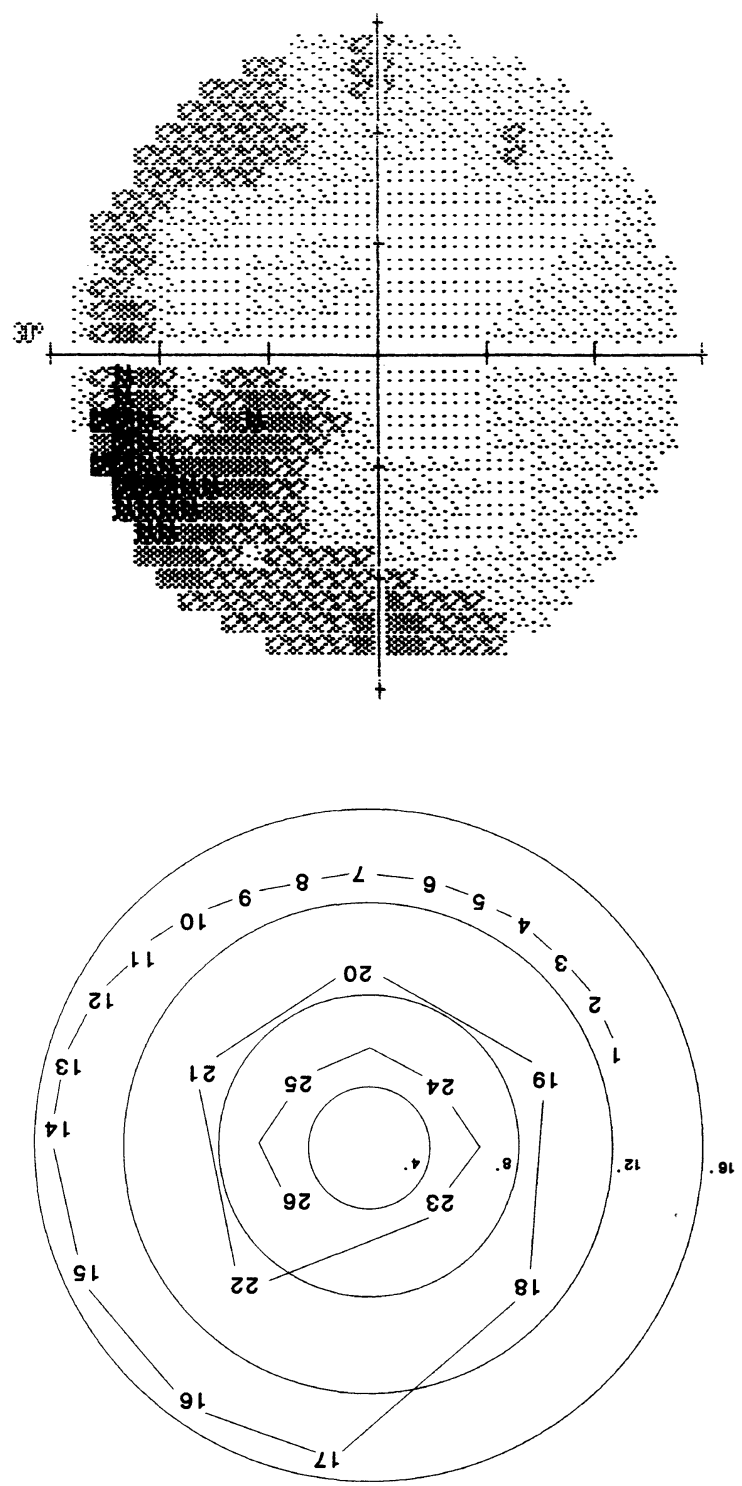

Fig. 5. False negative OKP: grey scale HVFA reveals inferior nasal scotoma, but OKP of the same eye (below) is normal.

revealed defects in one or both eyes, and therefore these OKP tests were true positives (Figs. 3 and 4). In the other 9 patients the positive OKP result was deemed a false positive as HVFA failed to detect any defect in the quadrant of the field in which OKP had detected a defect. Of these 9 patients, HVFA showed 5 patients to have entirely normal fields in both eyes, and in the other 4 HVFA did reveal a significant field defect but in a quadrant judged normal by the OKP test. Although these 4 patients were in fact glaucomatous, OKP did not reveal their glaucomatous defects and the defects it indicated were in an area of normal field. The OKP results are thus false positives and they are included as such in the analysis.

In the 21 patients with negative (i.e. normal) OKP tests, HVFA showed 8 to have normal visual fields, and in these 8 the OKP results were therefore 'true' negatives. In the other 13 patients these results were false negatives as HVFA showed glaucomatous field loss (Fig. 5). In 11 of 


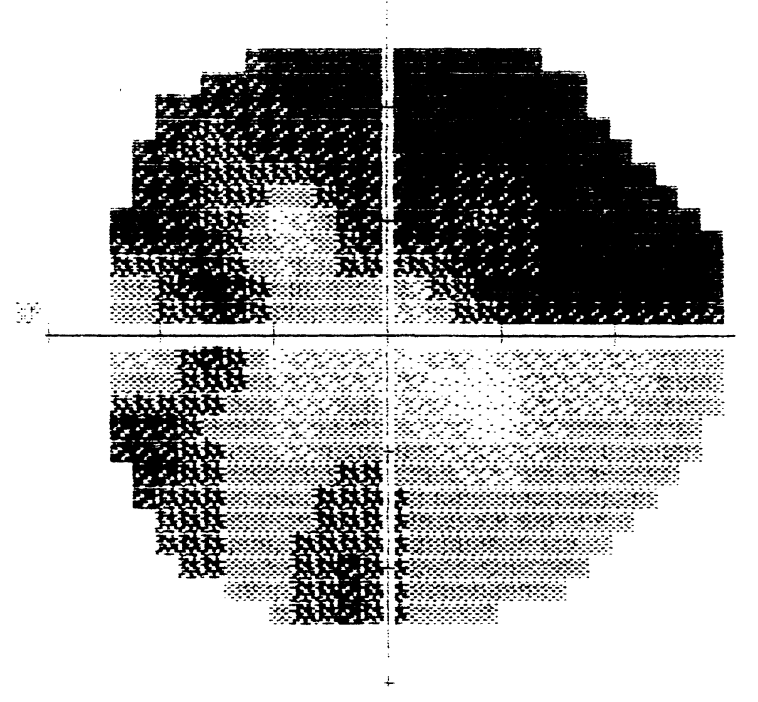

negatives) +4 (false positives, in which OKP missed a glaucomatous defect, but also gave a false positive result in another quadrant of the field) $] \times 100=60.5 \%$. The specificity is given by the number of normals identified by $\mathrm{OKP}$ as a percentage of the total number of normals and is $61.5 \%$, calculated as follows: 8 (true negatives) $\div[8+5$ (false positives) $] \times 100=61.5 \%$.

In the 26 true positive patients a total of between one and 18 of the 26 numbers on the OKP chart were missed; in 11 patients $(40 \%)$ between one and three numbers only were missed. In the patients with false positive OKP results, only one OKP test showed a defect of more than three points.

\section{Nature of Glaucoma by Clinical Examination}

Clinical examination of the 26 true positive patients revealed a diagnosis of open angle glaucoma (OAG) in 21 , low tension glaucoma (LTG) in 1, and chronic angle closure glaucoma (CAGG) in 4. Biomicroscopy of the
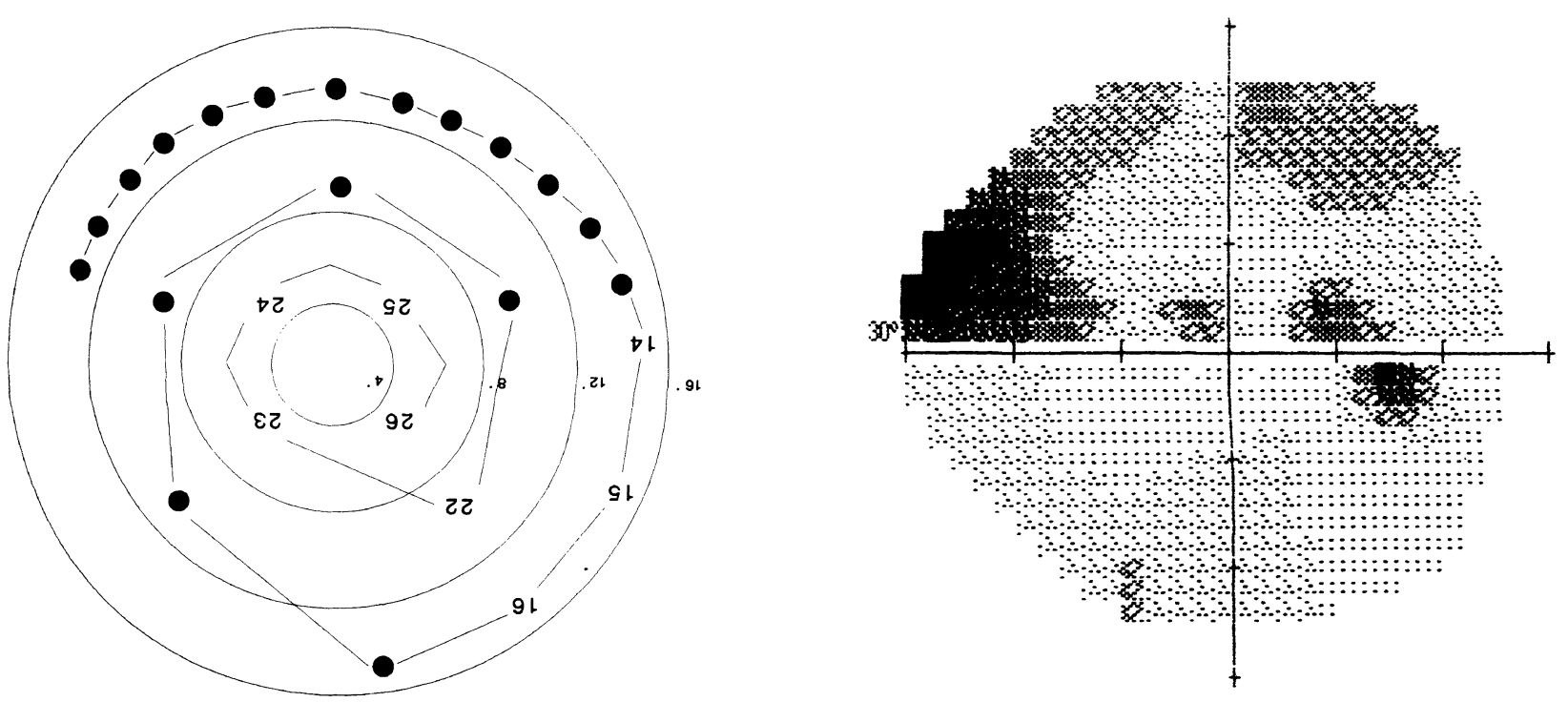

Fig. 6a.

Fig. 6. Left (a) and right (b) HVFA grey scales of patient whose optic discs are shown in Fig. 2. There is good correlation of the left OKP with the HVFA grey scale above, but the right OKP fails to detect a dense nasal step shown in the right HVFA grey scale.

these false negative patients the visual field loss detected by HVFA and missed by OKP lay within the area of the field tested by OKP. In addition, in these 11 patients the OKP test commonly missed a glaucomatous defect lying in the nasal step area between $15^{\circ}$ and $25^{\circ}$ - an area that lies outwith the area tested by OKP (Fig. 6). In the other 2 patients this was the only area of visual field abnormality. Table II gives the age and sex distribution of patients with reference to each subgroup.

The sensitivity of the test is given by the number of patients with glaucoma it identified as a percentage of the total number of patients with glaucomatous visual field loss identified by HVFA. Thus the sensitivity is $60.5 \%$, calculated as follows: 26 (true positives) $\div[26+13$ (false

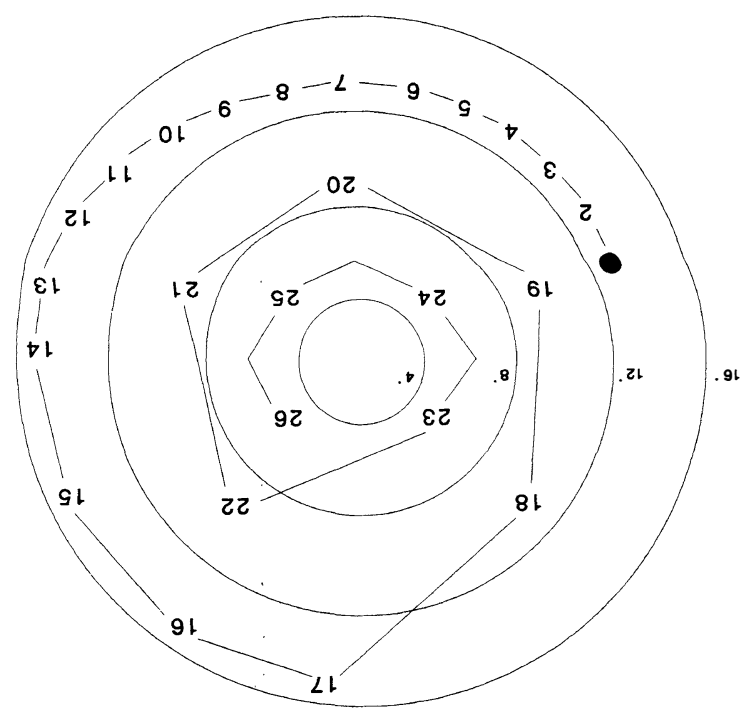

Fig. 6b. 
Table III. Correlation between number of defects on OKP and field loss identified by HVFA in the 26 true positive patients ( 36 abnormal OKP fields). The average HVFA mean deviation (MD) and corrected pattern standard deviation (CPSD) are given in decibels (dB) plus standard deviation

\begin{tabular}{lcrr}
\hline $\begin{array}{l}\text { No. of defects } \\
\text { on OKP chart }\end{array}$ & $\begin{array}{c}\text { No. of } \\
\text { fields }\end{array}$ & MD & \multicolumn{1}{c}{ CPSD } \\
\hline 1 & 7 & $-3.5+1.77$ & $3.6+1.1$ \\
2 & 6 & $-5.52+3.5$ & $8.1+3.9$ \\
3 and 4 & 6 & $-6.6+4.6$ & $6.6+5.3$ \\
5 to 10 & 8 & $-13.2+8.0$ & $8.8+4.4$ \\
11 to 18 & 9 & $-15.0+6.9$ & $10.8+3.4$ \\
\hline
\end{tabular}

optic discs in the worse eye of these 26 patients showed advanced glaucomatous damage in 18 discs: in 9 there was bean-pot cupping and in 9 loss of more than 3 clock hours of inferior and/or temporal neuroretinal rim. In 5 eyes the glaucomatous disc damage was less severe, with disc asymmetry, vertical elongation of cup, undermining of rim edge, pallor or thinning of the neuroretinal rim. Three patients with true positive results on OKP (defects corresponding to HVFA defects) had optic discs judged normal by clinical examination.

Of the 13 false negative patients, 11 had a clinical diagnosis of glaucoma with obvious optic disc pathology and a clinical history of low tension glaucoma (1 patient), chronic angle closure glaucoma (3 patients) and chronic open angle glaucoma ( 7 patients). Clinical history and examination of the optic discs of the 2 remaining false negative patients with defects confined to the nasal step area on HVFA revealed a diagnosis of ocular hypertension in that the patients had persistently raised intraocular pressure and no obvious evidence of optic disc pathology.

\section{Nature of Visual Field Loss}

The field loss identified by OKP in the 26 true positive patients was studied to determine the relationship between an OKP defect and a HVFA defect. In these 26 patients 36 eyes had defects on OKP corresponding to HVFA defects. For these 36 eyes the number of points missed on OKP was correlated with mean deviation (MD) from normal

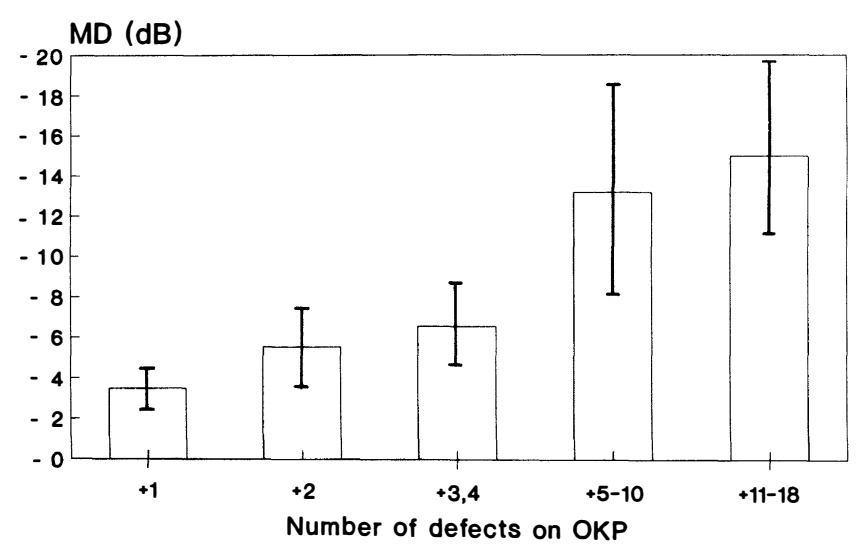

and the corrected pattern standard deviation (CPSD) as calculated for each visual field by Statpac 2 of the HVFA. Table III shows the relationship between number of points missed on OKP and the average MD and CPSD of the HVFA in these 36 eyes. In the 13 patients with false negative OKP tests the average visual field loss detected by HVFA was MD $-5.92+4.12 \mathrm{~dB}$ and CPSD $6.2+3.34 \mathrm{db}$ (mean plus standard deviation). The results for the true positive patients are shown graphically in Fig. 7.

To determine the defect depth in decibels that OKP can detect, the OKP fields of the more severely affected eye of the 26 true positive patients were examined and the minimum decibel defect detected by HVFA was recorded which corresponded to an area of OKP defect. The range of defects detected by HVFA was from 8 to $33 \mathrm{~dB}$ (mean $20.8 \mathrm{~dB}$ ) at points corresponding to the OKP defects. However, in $13(50 \%)$ of these patients, HVFA had detected defects in another quadrant of the field judged normal by OKP (Figs. 3 and 4). This loss undetected by OKP ranged from 9 to $30 \mathrm{~dB}$ (mean $19.6 \mathrm{~dB}$ ). In 13 patients with bilateral HVFA loss, OKP was positive in both eyes of 9 patients, but in the other 4 patients was positive only in the more severely affected eye (Fig. 8).

Fourteen patients had advanced field loss which can be described as 'split-fixation' in that at least one of the four innermost points on the Humphrey visual field was involved by glaucomatous loss ${ }^{10}$ contiguous with loss of almost an entire quadrant of field. Despite this degree of central visual field loss, the OKP charts of 10 of these 14 patients' visual fields were normal within the central $8^{\circ}$ tested (i.e. OKP numbers 23 to 26 all seen). Figs. 4 and 6 show typical examples of this.

\section{DISCUSSION}

This study set out to evaluate the sensitivity and specificity of OKP in detecting glaucomatous visual field loss. Automated perimetry with the HVFA was used to evaluate OKP's ability to identify the presence of field loss in patients and also to quantify the nature of any field loss

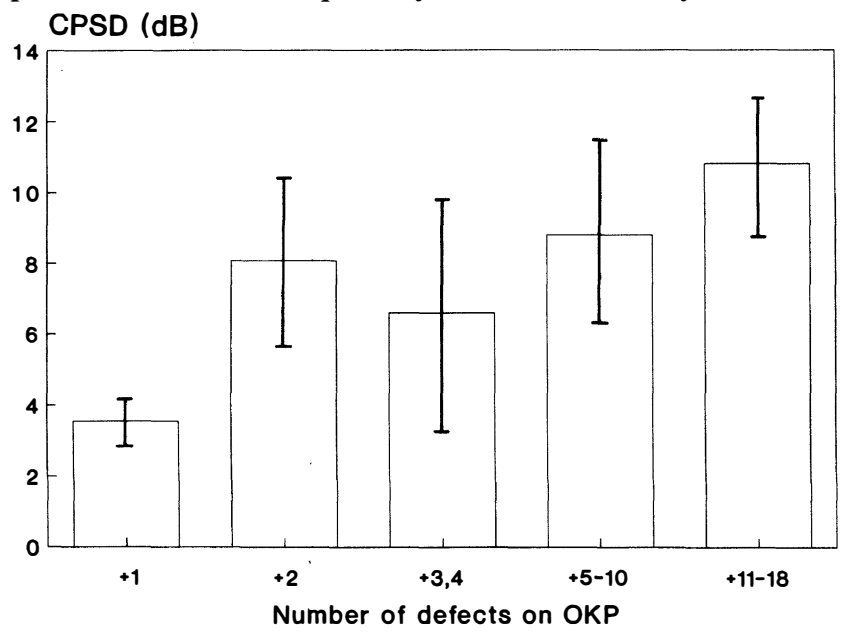

Fig. 7. Average HVFA mean deviation (MD) in decibels $(\mathrm{dB})$ (+ standard deviation) against number of defects (points missed) detected by OKP. (b) Average HVFA corrected pattern standard deviation (CPSD) in decibels ( + standard deviation) against number of defects (points missed) detected by OKP. 

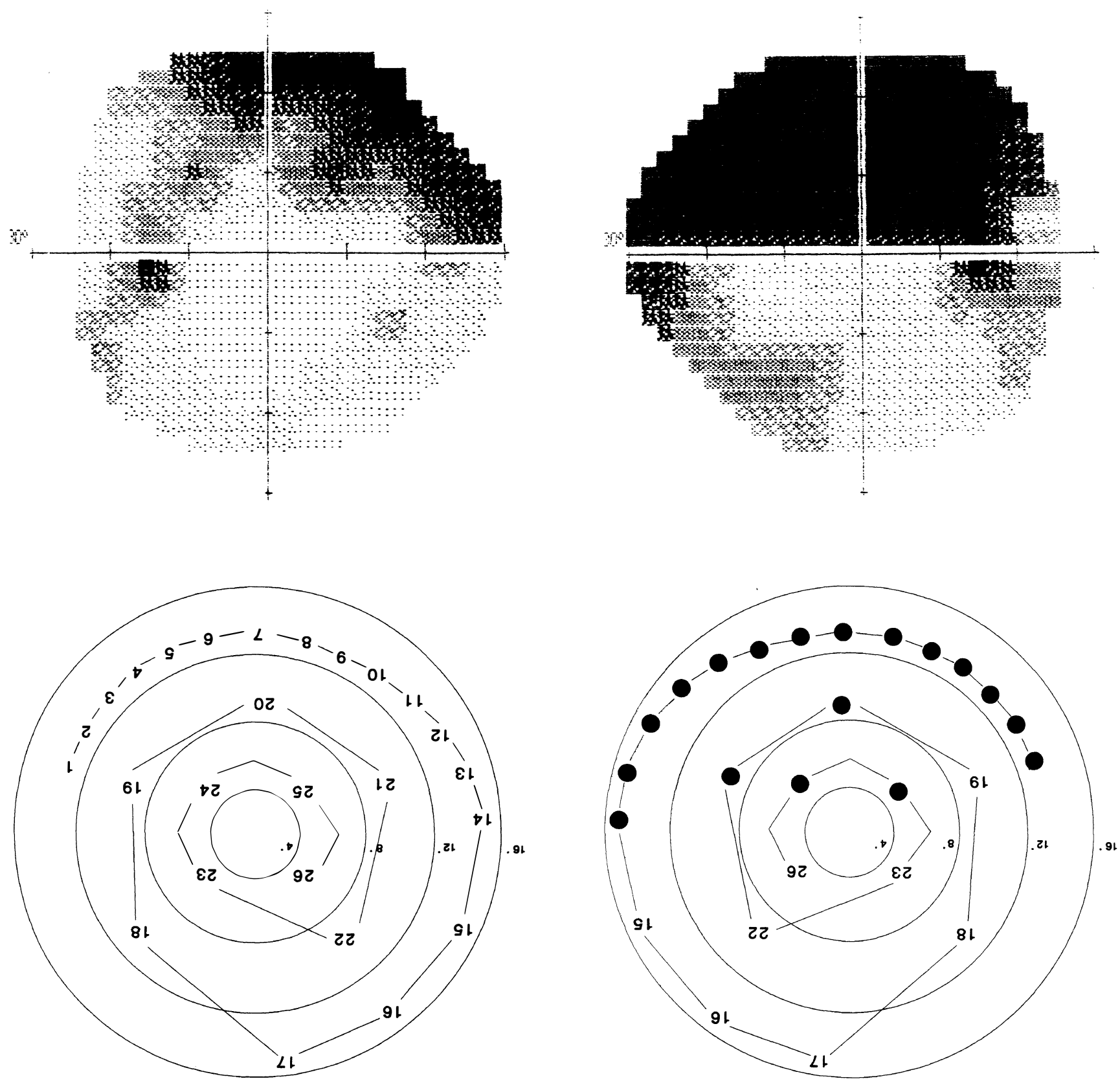

Fig. 8. Left (a) and right (b) HVFA grey scales with corresponding OKP charts below in a true positive patient. Although the right OKP (b) detected field loss, in the left eye the OKP was normal (a) despite the presence of a dense upper arcuate scotoma shown on HVFA.

detected. Automated perimetry provides an objective, reliable and repeatable method of detecting visual field loss, and has considerable advantages over manual methods. It is more sensitive than manual perimetry and therefore detects visual field loss at an earlier stage. ${ }^{12-16}$ It provides standardised technique, therefore freeing the results from operator error or observer bias. ${ }^{13,14}$ It gives internal reliability measurements of the patient's responses and thresholding is carried out using agecorrected normal data. ${ }^{7}$ The computer software also provides appropriate statistical analysis of data, relieving the clinician of the necessity of laboriously examining all the numerical data presented. ${ }^{7}$ In particular, use of the Glaucoma Hemifield Test with HVFA provides an objective and reliable assessment of whether a defect signifies the presence of glaucoma. ${ }^{17}$ HVFA therefore provides an objective and highly sensitive demonstration of the normality or otherwise of a visual field for comparison with OKP test results.

In this study OKP was found to have a sensitivity of $60.5 \%$ and a specificity of $61.5 \%$ for the detection of glaucomatous visual field loss. In addition, this study showed that the average decibel defect detected by OKP was $20.8 \mathrm{~dB}$. Quigley has shown that if automated perimetry shows a $10 \mathrm{~dB}$ deficit, then $40 \%$ of the normal retinal ganglion cells have been lost from that retinal point. ${ }^{18}$ OKP therefore seems capable of detecting only advanced glaucomatous damage. It is also significant that although OKP detected on average $20.8 \mathrm{~dB}$ loss in the 26 true positive patients, in 13 of those an average loss of $19.6 \mathrm{~dB}$ was 
missed in another quadrant. Although OKP is confined to only the central $16^{\circ}$ of the visual field, the results show that it was least accurate in detecting field loss within the central $8^{\circ}$. HVFA showed that in 14 of the 26 true positive patients central field loss 'splitting fixation' was present, yet in only 4 patients was any defect identified by OKP within the central $8^{\circ}$ of the field. Such advanced central field loss, which in many cases was absolute, should have been identifiable by OKP. It may be that these central defects were either missed or underestimated by OKP because in refixating to find the next of the inner numbers within the central $8^{\circ}$, small fixation saccades may reveal the central target spot to the subject.

Only 1 patient in the 9 false positives had a chart with an OKP defect missing more than three numbers, and therefore if loss of less than three numbers was disregarded, only 1 patient would have had a false positive result - thus increasing the specificity of the test. However, disregarding a defect of less than three numbers on OKP would dramatically worsen the sensitivity of the test as $40 \%$ of the true positive results only missed between one and three numbers. HVFA showed the patients with false negative OKP results had an average field loss of MD $-5.92 \mathrm{~dB}$ and CPSD $6.2 \mathrm{~dB}$, which is equivalent to the true positive patients who had defects of between one and three numbers on OKP (Table III and Fig. 7).

OKP has been compared favourably with conventional perimetry in previous studies, but in these the comparative perimetry was performed using manual Oculus Tubinger perimetry for some patients, and Dicon 3000 two-zone suprathreshold testing in others. ${ }^{1,19}$ Modern threshold automated perimetry, with perimeters such as the Humphrey visual field analyser, has been shown to be superior to other perimetric techniques ${ }^{12-16}$ and this may explain why in the present study OKP did not compare so favourably.

The purpose of the present study was not to compare the efficacy of HVFA and OKP as screening techniques, but to use HVFA to provide knowledge of the precise nature, depth and location of scotomas that OKP can detect. The results show that OKP's sensitivity and specificity for the detection of field loss were low in a group of patients the majority of whom were suffering from glaucoma. In the glaucoma patients with moderate or severe optic disc damage OKP generally detected field loss and identified these individuals as glaucomatous. However, this finding must be tempered with the knowledge that such advanced disease is rare in the community, and therefore in any screening programme the number of normal individuals would greatly exceed the number of individuals with glaucomatous field loss. Among those tested who missed between one and three points on OKP such loss was equally likely to represent the presence of significant visual field loss or a false positive result. The fact that significant HVFA loss went undetected by OKP in the fellow, less affected eye of 4 of the 13 patients with bilateral field loss prompts the question as to whether the sensitivity of the test might have been even poorer had the population studied had less severe field loss. OKP's inability to detect field loss in the less severely affected eye of these 4 glaucoma patients, the fact that it missed scotomas of an average of $19.6 \mathrm{~dB}$ in a different quadrant from a field in which it detected an average defect of $20.8 \mathrm{~dB}$, and its inaccuracy in detecting advanced field loss splitting fixation, indicate that OKP persistently underestimates glaucomatous visual field loss. It is therefore not a suitable test for self-testing by glaucoma patients of their own visual field as has been proposed. ${ }^{2}$

It has been suggested that OKP may detect subtle visual field loss not detectable by conventional perimetric techniques, and Damato et al. cite the finding of a $41 \%$ rate of positive OKP tests in eyes with ocular hypertension, a suspicious disc or contralateral glaucoma in support of this conclusion. ${ }^{2}$ The results of the present study, however, show a near-linear correlation between the number of points missed on the OKP chart and the glaucomatous deficit measured by HVFA (Fig. 7). As the number of points missed on OKP increases, the mean deviation from normal (a measure of overall reduction in sensitivity of the field) increases. A similar pattern was also observed between the number of OKP defects and CPSD. The CPSD tends to be high in the presence of localised field loss, even if the overall MD is not significantly reduced. ${ }^{7}$ Thus OKP seems to detect both generalised and localised glaucomatous field loss, and it is therefore unlikely that some type of damage is being detected that is not identifiable by conventional perimetry.

To be effective, any screening programme must have a high sensitivity and specificity for the disease, as false negatives will mean an individual is wrongly reassured that he or she is disease-free. Any test that carries a high false positive rate will equally detract from the efficient use of resources and may, in the long-term, be counterproductive to the efficient delivery of limited ophthalmic services.

Optic disc analysis by a a trained observer using slitlamp biomicroscopy may be able to predict the absence of a field defect, ${ }^{10}$ but the resources are not normally available for this to be used in a community screening programme. Tonometry alone has been described as being 'worse than useless' in glaucoma screening programmes because of the false positive and false negative results it produces. ${ }^{3}$ Visual field analysis has been said to be the ideal method of screening for glaucoma, ${ }^{4}$ and automated perimetry has already been shown to be suited to this task. ${ }^{3.5 .12}$ The results of this study show that although OKP can detect advanced glaucomatous damage it would be of little value in a screening programme because of its low sensitivity and low specificity.

I would like to thank Mrs. C. Owen for technical and administrative assistance, and Mr. P. Bloor for help with the illustrations.

Key words: Field loss, Glaucoma, Perimetry.

\section{REFERENCES}

1. Damato BE. Oculo-kinetic perimetry: a simple visual field 
test for use in the community. Br J Ophthalmol 1985;69: 927-31.

2. Damato BE, Chyla J, McClure E, Jay JL, Allan D. A handheld OKP chart for the screening of glaucoma: preliminary evaluation. Eye 1990;4:633-7.

3. Mundorf TK, Zimmerman TJ, Nardin GF, Kendall KS. Automated perimetry, tonometry, and questionnaire in glaucoma screening. Am J Ophthalmol 1989;108:505-8.

4. Armaly MF. Ocular pressure and visual fields. Arch Ophthalmol 1969;18:25-40.

5. Kosko O, Sommer A, Auer C. Screening with automated perimetry using a threshold related three-level algorithm. Ophthalmology 1986;93:882-6.

6. Statpac 2 Owner's Manual. San Leandro, California: Allergan Humphrey, 1991.

7. Flammer J, Drance SM, Augustiny L, Funkhouser A. Quantification of glaucomatous visual field defects with automated perimetry. Invest Ophthalmol Vis Sci 1985;26: $176-81$.

8. Heijl A, Lindgren G, Lindgren A et al. Extended empirical statistical package for evaluation of single and multiple fields in glaucoma: Statpac 2. In: Mills RP, Heijl A, editors. Perimetry update 1990/91. Proceedings of the IX International Perimetric Society Meeting; New York: Kugler Publications, 1991:303-15.

9. Anderson DR. In: Perimetry with and without automation. St Louis: Mosby, 1987:233

10. Hoskins HD Jr, Gelber SC. Optic disc topography and visual field defects in patients with increased intraocular pressure. Am J Ophthalmol 1975;80:284-95.

11. Liebermann MF, Ewing RH. Reassessing split fixation in advanced glaucoma: preliminary studies with computerized perimetry. In: Mills RP, Heijl A, editors. Perimetry update
1990/91. Proceedings of the IX International Perimetric Society Meeting; New York: Kugler Publications; 1991: 473-89.

12. Heijl A. Automatic perimetry in glaucoma visual field screening: a clinical study. Graefes Arch Clin Exp Ophthalmol 1976;200:21-37.

13. Azuma I, Tokuoka S. The usefulness of automated perimetry in detecting early glaucoma. In: Greve EL, Heijl A, editors, seven International Visual Fields Symposium. Doc Ophthalmol Proc Ser, Dordrecht: Nijhoff/Junk, 1987;49: 391-6.

14. Beck RW, Bergstrom TJ, Lichter PR. A clinical comparison of visual field testing with a new automated perimeter, the Humphrey Field Analyzer, and the Goldmann perimeter. Ophthalmology 1985;92:77-82.

15. Hotchkiss ML, Robin AL, Quigley HA, Pollack IP. A comparison of Peritest automated perimetry and Goldmann perimetry. Arch Ophthalmol 1985;103:397-403.

16. Mills RP, Hopp RH, Drance SM. Comparison of quantitative testing with the Octopus, Humphrey and Tubinger perimeters. Am J Ophthalmol 1986;104:496-504.

17. Katz J, Sommer A, Gaasterland DE, Anderson DR. Comparison of analytic algorithms for detecting glaucomatous visual field loss. Arch Ophthalmol 1991;109:1684-9.

18. Quigley HA, Dunkelberger GR, Green WR. Retinal ganglion cell atrophy correlated wth automated perimetry in human eyes with glaucoma. Am J Ophthalmol 1989;107: 453-64.

19. Alvarez E, Damato BE, Jay JL. Comparative evaluation of oculokinetic perimetry and conventional perimetry in glaucoma. Br J Ophthalmol 1988;72:258-62. 